\title{
Chickenpox Chorioretinitis with Retinal Exudates and Periphlebitis
}

\author{
Hirokuni Kitamei $^{a} \quad K^{2}$ ichi Namba ${ }^{a}$ Nobuyoshi Kitaichi ${ }^{b, c}$ \\ Akiko Wakayama $^{a}$ Shigeaki Ohno ${ }^{b}$ Susumu Ishida ${ }^{a}$ \\ Departments of ${ }^{\mathrm{a} O p h t h a l m o l o g y}$ and ${ }^{\mathrm{b}}$ Ocular Inflammation and Immunology, \\ Hokkaido University Graduate School of Medicine, and ' Department of \\ Ophthalmology, Health Sciences University of Hokkaido, Sapporo, Japan
}

\section{Key Words}

Chickenpox · Chorioretinitis - Intraocular inflammation · Primary VZV infection . Uveitis · Varicella zoster virus · Acute retinal necrosis

\section{Abstract \\ Background: Chickenpox is rarely associated with posterior segment inflammation. We report on a case of unilateral chickenpox chorioretinitis with retinal exudates and periphlebitis.}

Case Presentation: A 21-year-old healthy man, who suffered from chickenpox 2 weeks prior to symptom development, exhibited mild anterior chamber cells, vitreous opacity, sheathing of retinal veins, and yellow-white exudates in his right eye. Varicella zoster virus DNA was detected by polymerase chain reaction in the aqueous humor. He was treated with intravenous acyclovir followed by oral prednisolone and valaciclovir. Aqueous cells quickly disappeared and retinal exudates diminished within 1 month, leaving faint retinal scarring. Retinal arteritis had never been observed in this patient.

Conclusions: Although the ocular findings in this case were similar to acute retinal necrosis (ARN), the clinical features differed from ARN in the following points: (1) mild anterior chamber inflammation, (2) absence of retinal arteritis, and (3) prompt resolution of inflammatory findings. The distinctive clinical features indicated that chorioretinitis associated with chickenpox may not have the same pathological conditions as ARN.

\section{Introduction}

Varicella zoster virus (VZV) causes chickenpox at primary infection among children and young adults. After a long incubation period in sensory nerve ganglia, VZV is reactivated and causes herpes zoster and a wide range of neurological diseases [1]. 
Ocular inflammations caused by reactivation of VZV include keratitis, iridocyclitis, and acute retinal necrosis (ARN) [2].

Chickenpox, caused by primary VZV infection, is infrequently associated with ocular inflammation, including conjunctivitis, keratitis, and iridocyclitis, and rarely with posterior segment inflammation. Here, we report on a rare case of chorioretinitis complicated with chickenpox and discuss the dissimilarity to ARN by referring to the previous literature.

\section{Case Report}

This study was performed with informed consent and followed the guidelines of the Ethics Committee of the Hokkaido University Graduate School of Medicine. A 21-year-old healthy man visited an ophthalmology clinic complaining of floaters and ocular hyperemia in his right eye. Since he was suspected to develop ARN, he was referred to our hospital on the same day.

The patient suffered from chickenpox 2 weeks prior to his ocular symptoms. Fever had already diminished and skin eruptions changed to crusts without medication.

At the first visit, his visual acuity was $20 / 20$ and the intraocular pressure was $18 \mathrm{~mm} \mathrm{Hg}$ in both eyes. In the right eye, slit-lamp examination detected ciliary injection, $3+$ cells in the anterior chamber, mutton-fat keratic precipitates, and Koeppe's iris nodules. Fundus examination revealed slight vitreous opacity, sheathing of retinal veins, and yellow-white exudates. The exudates of 1-3 disc diameters were well demarcated and scattered in all quadrants of the peripheral retina ( $\underline{\text { fig. } 1}$, fig. $2 \mathrm{a}, \mathrm{b}$ ). Fluorescein angiography showed no leakage from the optic disc and retinal vessels and no avascular area (fig. 2c). His left eye showed no symptoms of inflammation.

Both serum anti-VZV immunoglobulin $\mathrm{M}$ and immunoglobulin $\mathrm{G}$ antibodies were elevated to 3.7 M.I. and 21.0 G.I., respectively (cut-off level 0-0.9). VZV DNA was detected by polymerase chain reaction from the aqueous humor.

The patient was immediately treated with continuous drip infusion of acyclovir ( $30 \mathrm{mg} / \mathrm{kg} / \mathrm{day}$ ) for 1 week and oral valaciclovir (20 mg/kg/day) for the following 2 months as well as oral prednisolone $(0.6 \mathrm{mg} / \mathrm{kg} / \mathrm{day})$, starting 8 days after acyclovir treatment began.

Aqueous inflammatory cells disappeared within a few days. Retinal exudates also shrank immediately and diminished within 1 month, leaving faintly pigmented retinal scarring. Sheathing of retinal veins also disappeared without abnormal changes. The patient has preserved $20 / 20$ or better visual acuity for the following 2 years and has remained free from intraocular inflammation.

\section{Discussion}

One of the issues in this case is whether we can call the observed disorder 'ARN' or not. ARN is diagnosed according to the criteria of the Executive Committee of the American Uveitis Society and is characterized by peripheral necrotizing retinitis, retinal arteritis, and a prominent inflammatory reaction in the vitreous and anterior chamber [3]. In the present case, the following two findings were incompatible with these criteria: (1) sheathing of vessels was observed in the retinal veins but not in the arteries, and (2) intraocular inflammation in the anterior segment was mild.

The complication of chorioretinitis with chickenpox has been reported using different diagnostic terms such as 'ARN with chickenpox', 'chickenpox chorioretinitis' or 'varicella chorioretinitis' [4,5]. Recently, Tajunisah and Reddy [6] reported such a case and reviewed the literature of 16 cases of chorioretinitis following chickenpox, 
although they used the term 'ARN complicating chicken pox'. In the majority of these cases, the inflammation was mild and less fulminant with good visual prognosis, and retinal detachment with multiple retinal breaks in the area of exudates never occurred.

Systemic pathological response is immunologically different between primary and reactivated infection of VZV, suggesting that inflamed eyes may show different clinical features. Compared with ARN (a local infection with reactivated VZV), chickenpox (a primary infection with VZV) has higher anti-varicella immune response which may contribute to prompt improvement of ocular inflammation with good visual prognosis. Furthermore, the fact that retinal lesions with exudates hardly cause retinal breaks in chickenpox indicates that pathological changes may not be 'necrotic' in the lesions. Therefore, it may not be accurate to diagnose chorioretinitis complicated with chickenpox as 'ARN'. In order to establish the adequate nomenclature, further accumulation of similar cases is needed.

The present patient complained of only mild symptoms such as slight floaters and hyperemia. Though this case was an adult man, chickenpox commonly affects children. Young children may not complain of these mild symptoms, even if they suffer from chickenpox chorioretinitis. Therefore, both pediatricians and ophthalmologists should pay attention to the ocular condition in children with chickenpox.

\section{Disclosure Statement}

None of the authors have a conflict of interest to disclose. 


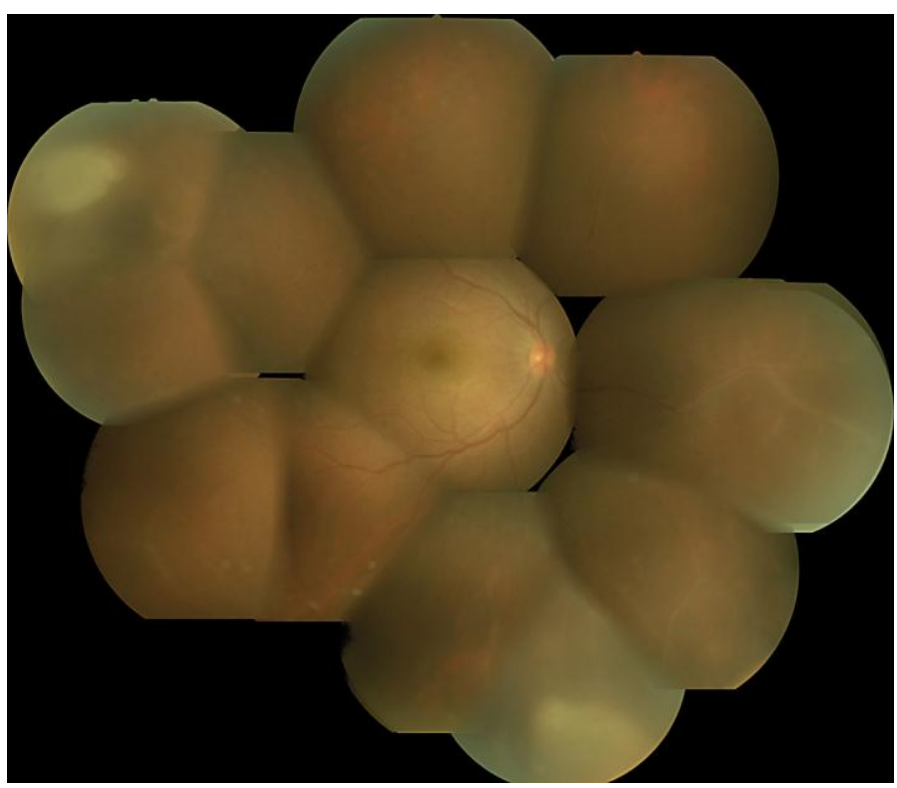

Fig. 1. Fundus photograph of the right eye before treatment. At the first examination, vitreous opacity, sheathing of retinal veins, and yellow-white exudates were observed. The exudates were well demarcated and presented in the peripheral retina and not connected with each other. 

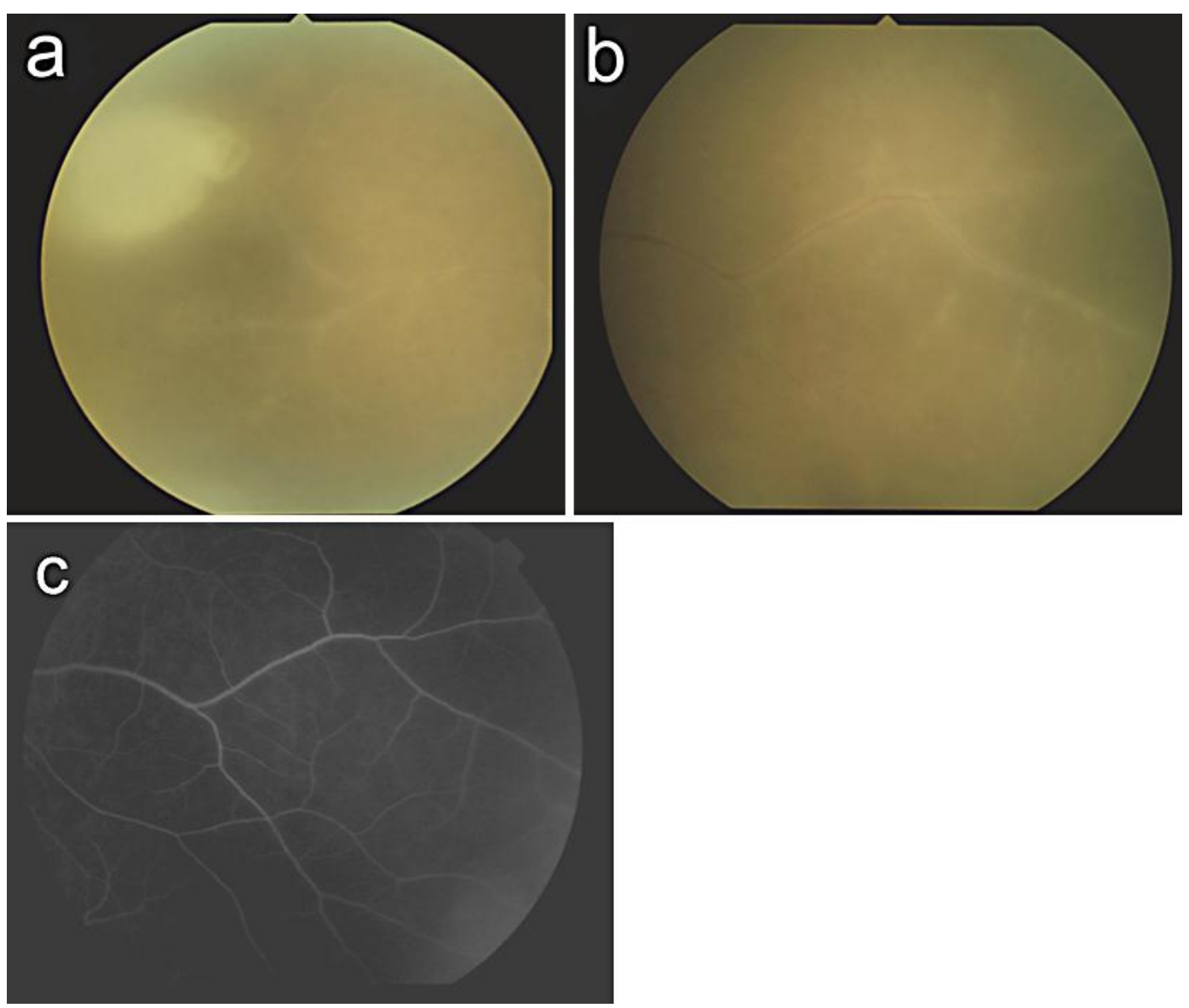

Fig. 2. Higher magnification photographs showed a well-demarcated exudate in the superotemporal retina (a) and sheathing of retinal veins in the nasal retina (b). Of note is that sheathing was observed in the retinal veins but not in the arteries. Fluorescein angiography showed no fluorescein leakage from the veins $(\mathbf{c})$.

\section{References}

1 Mueller NH, Gilden DH, Cohrs RJ, Mahalingam R, Nagel MA: Varicella zoster virus infection: clinical features, molecular pathogenesis of disease, and latency. Neurol Clin 2008;26:675-697.

-2 Soushi S, Ozawa H, Matsuhashi M, Shimazaki J, Saga U, Kurata T: Demonstration of varicella-zoster virus antigens in the vitreous aspirates of patients with acute retinal necrosis syndrome. Ophthalmology 1988;95:1394-1398.

-3 Holland GN: Standard diagnostic criteria for the acute retinal necrosis syndrome. Executive Committee of the American Uveitis Society. Am J Ophthalmol 1994;117:663-667.

4 Kelly SP, Rosenthal AR: Chickenpox chorioretinitis. Br J Ophthalmol 1990;74:698-699.

-5 Aslan O, Soykan E, Ozkan SS: Varicella chorioretinitis. Acta Ophthalmol Scand 2007;85:907-908.

6 Tajunisah I, Reddy SC: Acute retinal necrosis complicating chicken pox in a healthy adult: a case report and review of literature. Ann Ophthalmol 2007;39:57-62. 